\title{
Navigierte Kyphoplastie
}

OP-Ablauf Normalerweise stellt die Therapie einer osteoporotischen Brustwirbelköperfraktur keine besondere Herausforderung für den Operateur dar. Die hier vorgestellte operative Behandlung einer osteoporotischen Kompressionsfraktur eines Brustwirbelkörpers ist jedoch nicht alltäglich: Die Patientin hatte eine extreme thorakolumbale Kyphoskoliose mit einem keilförmig deformierten zwölften Brustwirbelkörper, der gebrochen war und der Patientin starke Schmerzen bereitete.

- Andreas Draxinger -

Die Volkskrankheit Osteoporose ist eine häufige Ursache für Wirbelkörperbrüche, die operativ behandelt werden müssen. Die Osteoporose ist eine Erkrankung, die vor allem bei älteren Frauen nach der Menopause auftritt. Aufgrund der Abnahme der Knochendichte des gesamten Skeletts wird der Knochen insgesamt anfälliger für Brüche. Die häufigsten Knochenbrüche infolge der Osteoporose, welche auch gleichzeitig auftreten können, sind

- hüftgelenksnahe Oberschenkelfrakturen (Schenkelhalsfraktur),

- handgelenksnahe Frakturen (distale Radiusfraktur),

- Oberarmkopfbruch (subcapitale Humerusfraktur),

- Wirbelkörperfrakturen oder

- Beckenbrüche.

In der Regel wird die Vertebroplastie oder die Kyphoplastie als operative Maßnahme bei osteoporotischen Wirbelkörperfrakturen angewendet.

Die Vertebroplastie ist ein minimalinvasives chirurgisches Verfahren zur Therapie von Wirbelkörperfrakturen. Vor zirka 30 Jahren wurde mit dieser Therapie in Frankreich begonnen.

Üblicherweise wird unter Vollnarkose eine Jamshidinadel (Hohlnadel) über den Pedikel (Bogenwurzel) in den Wirbelkörper eingebracht. Anschließend wird Knochenzement in den Wirbelkörper gespritzt. Nachdem der Knochenzement ausgehärtet ist, stützt das Zement-Spongiosagebinde die Wirbelkörperstrukturen. Die meisten Patienten sind kurz darauf beschwerdefrei.

Die Kyphoplastie ist die weiter entwickelte Therapieform der Vertebroplastie und ist mittlerweile ein standardisiertes und bewährtes minimalinvasives chirurgisches Verfahren bei der Versorgung von osteoporotischen Kompressionsfrakturen der Wirbelkörper. Anhand von Studien konnte eine hohe Sicherheit und Wirksamkeit dieser Therapie belegt werden.

Das Ziel dieser Therapieform ist eine Wiederaufrichtung der Wirbelkompressionsfraktur und die Wiederherstellung der vorherigen Krümmung der Wirbelsäule (vorherige sagittale Ausrichtung) mithilfe eines Ballon-Katheters. Über einen perkutanen Knochenzugang wird der Ballon exakt positioniert und kontrolliert aufgedehnt. Die Ballon-Aufdehnung erfolgt über eine Spritze - die vorher luftfrei mit Kontrastmittel gefüllt wurde - und unter ständiger Röntgenkontrolle.

Die Aufdehnung des Ballons bewirkt so eine Zerquetschung (Kompaktierung) der Spongiosa und gleichzeitig so weit wie möglich eine Wiederaufrichtung der Wirbelkörperfraktur. In den Hohlraum wird anschließend Knochenzement eingebracht.

Bei Patienten, die nicht auf diese Weise operativ versorgt werden, ist häufig ein sogenannter Witwenbuckel zu sehen. Infolge des Krankheitsverlaufs mit häufig nachfolgenden Wirbelkompressionsfrakturen, bei denen sich die Wirbel wie ein Keil formen, kommt es zu einer verstärkten Verkrümmung der Wirbelsäule nach vorne (Kyphosierung), die mit einem Größenverlust des Patienten einhergeht ${ }^{1}$.

\section{OP-Planung und OP-Vorbereitung}

Nach vorausgegangener Diagnostik stellte der Operateur bei der oben erwähnten 70-jährigen Patientin folgende Diagnose: osteoporotische Kompressionsfraktur BWK XII (AO: 52-A1.1.1). Beim OP-Aufklärungsgespräch empfahl der Operateur, eine Kyphoplastie und/oder eine Vertebroplastie, um Schmerzfreiheit zu erreichen beziehungsweise ein weiteres Einbrechen des Wirbelkörpers zu verhindern. Die Patientin willigte daraufhin in die vorgeschlagene Therapie ein.

Der Operateur entschied sich, keine konventionellen Röntgengeräte einzusetzen, weil bei normalen Röntgenaufnahmen die Wirbelsäulenstrukturen aufgrund der Osteoporose nicht ausreichend genug darstellbar und abgrenzbar waren $(\rightarrow$ Abb. 1 und 2). Stattdessen wählte das Operationsteam den Einsatz des O-arm-Röntgenbildgebungssystems ( $\rightarrow$ Abb. 3), welches fluoroskopische 2-D- und 3-D-Bildaufnahmen ermöglicht und das Navigationssystem Stealth Station S7 der Firma Medtronic $(\rightarrow$ Abb. 4), um so die höchste Sicherheit für die Patientin zu gewährleisten.

Nach der Narkoseeinleitung und Freigabe der Patientin durch den Anästhesisten erfolgte die Lagerung standardisiert in Bauchlage auf einem Carbon-OP-Tisch mit speziellen Bauchlagerungskissen. Erforderliche Instrumente und OP-Einmalartikel wurden auf dem Instrumentier- und Beistelltisch vorbereitet. Anschließend wurde röntgengestützt die Region auf der Haut markiert, wo sich der gebrochene Wirbelkörper befand und Röntgenpositionen für die fluoroskopischen 2-D- und 3-D-Bildaufnahmen geplant beziehungsweise gespeichert.

Der Vorteil beim O-arm ist, dass vier Röntgenpositionen und eine Parkposition $(\rightarrow$ Abb. 5) mithilfe einer Memory-Funktion abgespeichert werden können. In der Parkposition schwenkt der O-arm nach kopfwärts, um für den Operateur und weitere OP-Beteiligte Platz zu schaffen, wenn nötig. Auch das Navigationsgerät wur- 
de positioniert und die Kamera so ausgerichtet, dass sie alle nötigen Navigationsinstrumente erfassen konnte ( $\rightarrow$ Abb. 6).

Nachdem die Neutralelektrode korrekt auf die Haut geklebt wurde und eine ausreichende Hautdesinfektion des OP-Gebiets erfolgte, wurde kontrolliert, ob die
Patientin trocken auf der Patientenauflage lag. Dann wurde die Abdeckung des OPGebiets und des O-arms mit sterilen Tüchern und einem speziellen O-arm-Bezug durchgeführt. Anschließend folgte das Anstecken des Chirurgiesaugers und des Monopolarhandstücks sowie deren Funkti- onskontrolle. Vor Hautschnitt führte das OP-Team das Team-Time-Out durch.

\section{OP-Ablauf}

Im ersten Schritt wurde mithilfe des Röntgens der erste Lendenwirbelkörper identifiziert und über den Dornfortsatz ein klei-

\section{1}
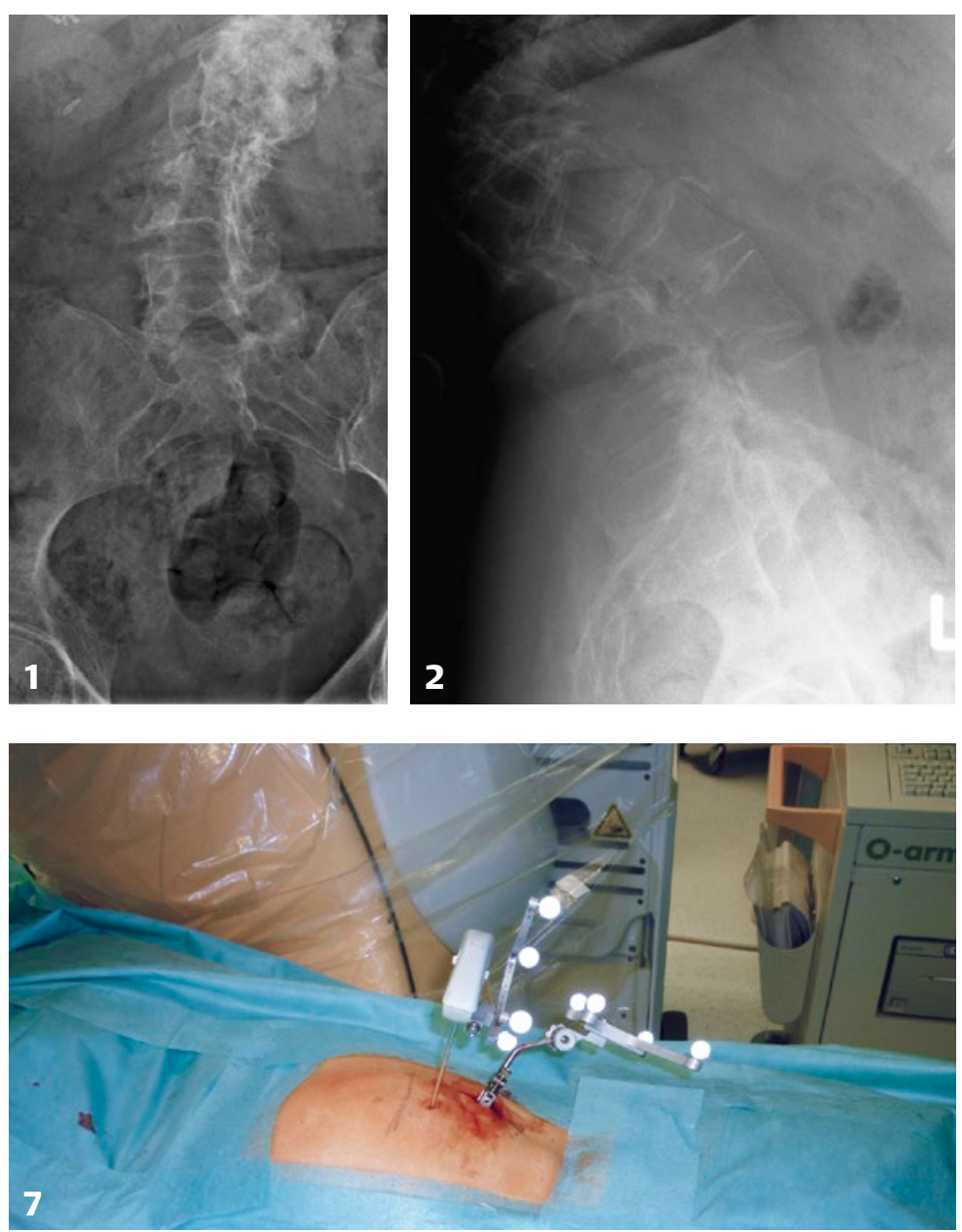

告

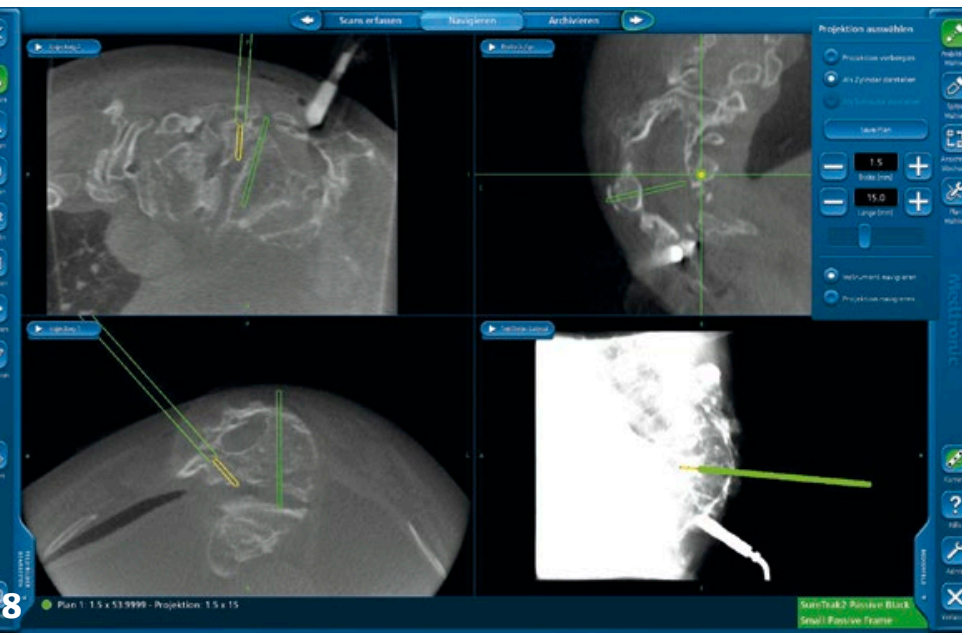

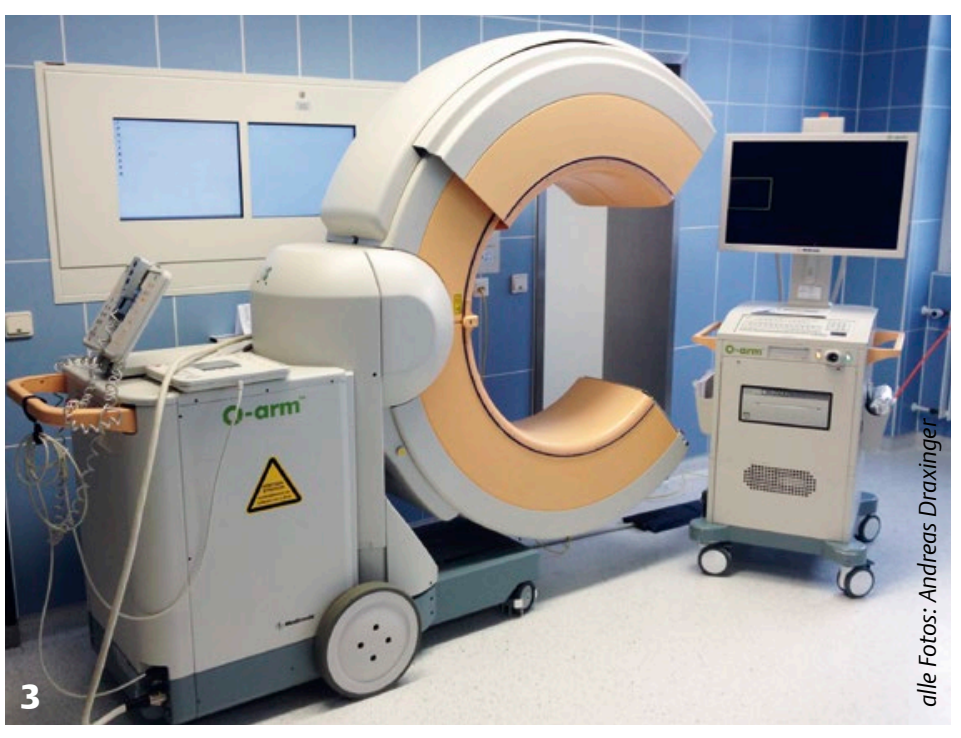

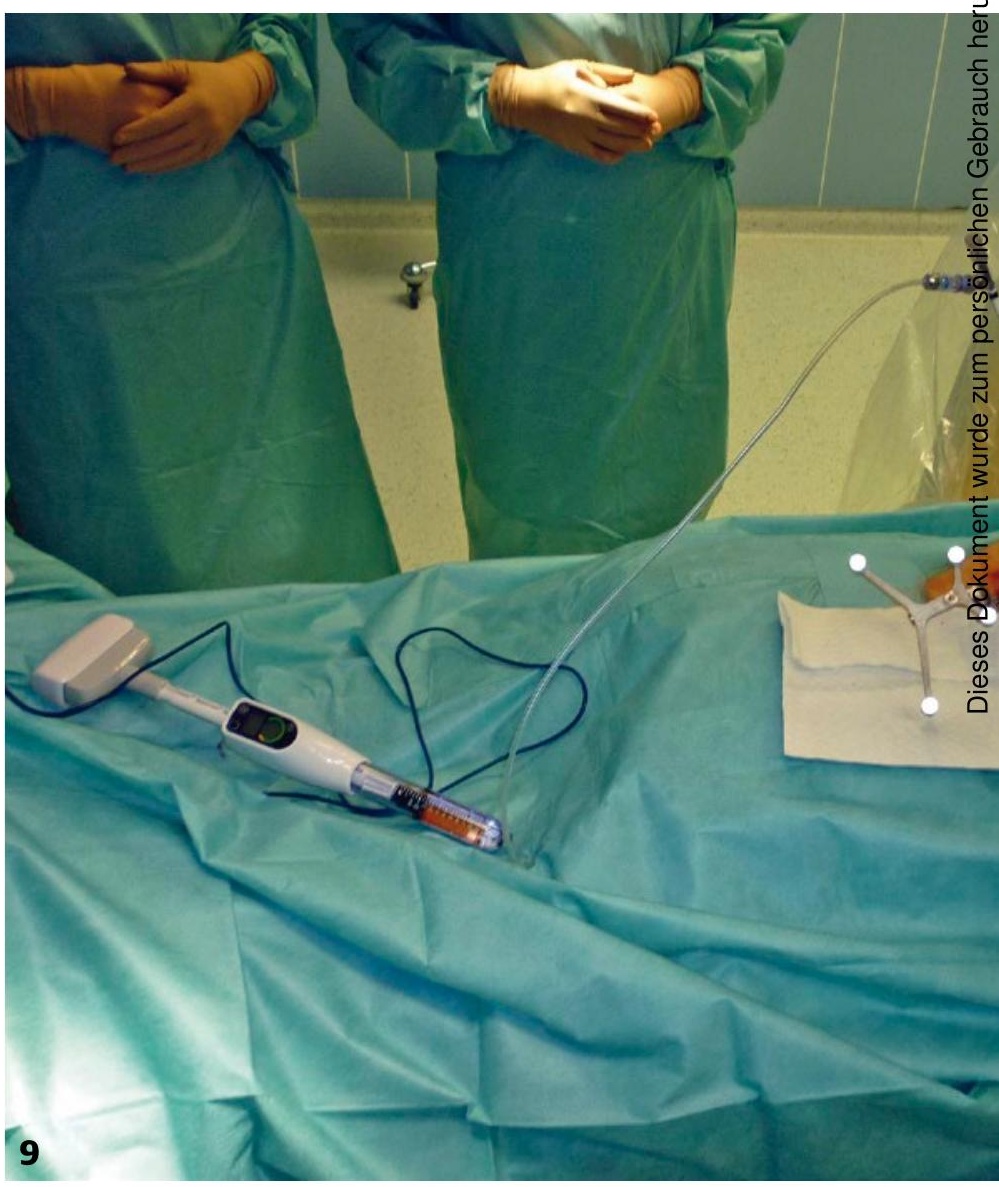


ner paramedianer Hautschnitt gesetzt. Es folgte die weitere Präparation bis auf den Knochen unter Längsspaltung der Faszie bilateral des Dornfortsatzes LWK1, das Verdrängen der autochthonen Rückenmuskulatur und das sichere Anklemmen der Wirbelsäulenklemme beim Dornfort- satz LWK1. Dann wurde die Wirbelsäulenklemme mit dem Referenzrahmen für die Navigation verbunden ( $\rightarrow$ Abb. 7), der eine dynamische Bezugnahme zur Anatomie der Patientin herstellte.

Zur Vorbereitung des 3-D-Scans wurde nochmals der Wirbelsäulenbereich TH11-
L1 in a.p. und seitlicher Röntgenkontrolle mit den Blenden (Kollimatoren) der Röntgenröhre eingegrenzt, um einen kollimierten Scan der Anatomie erfassen zu können und gleichzeitig die Streustrahlung im OPRaum und die Strahlenbelastung für die Patientin zu reduzieren.
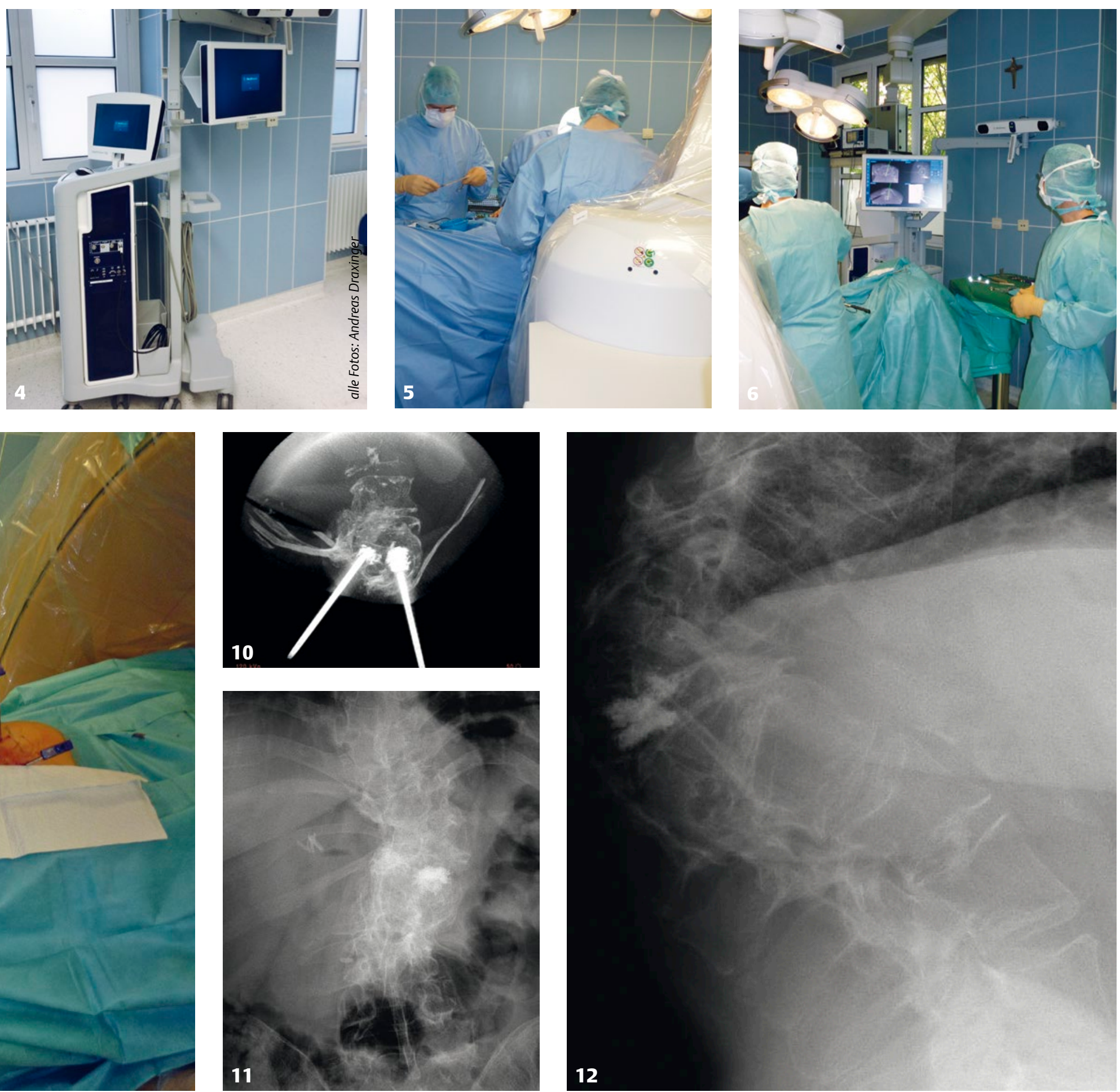
Im 3-D-Aufnahmemodus erstellte das O-arm ${ }^{\circledR}$-IAS dann eine Reihe von gepulsten Röntgenaufnahmen durch eine komplette Drehung des Gantry-Rotors um 360 Grad. Das System speicherte diese Aufnahmen und verwendete einen Rekonstruktionsalgorithmus, um daraus ein 3-D-Bild der Patientenanatomie zu entwickeln. Das Bild wurde dann auf dem MVS-Monitor in Hochauflösung in drei Ebenen (axial, koronal und sagittal) angezeigt. Gleichzeitig sendete das 0 -arm ${ }^{\circledR}$-IAS Bilddaten an die Stealth-Station-S7-Navigationseinheit.

Dann mussten die zur Operation benötigten Navigationsinstrumente angemeldet (verifiziert) und kalibriert werden. Das Verifizieren beziehungsweise Kalibrieren ist bei jeder OP erforderlich. Dieser Vorgang erfolgt immer nach den gleichen Arbeitsschritten, indem bestimmte Instrumente wie eine navigierbare Tastsonde (Pointer) in die Vertiefung des Referenzrahmens gehalten werden. Die Kamera der Navigation erkennt dann bestimmte Instrumente anhand der Form, auf der jeweils vier Einweg-Reflektormarker auf Sockel gesteckt werden.

Bei korrekter Durchführung ertönt ein bestimmter Glockenton. Bei dieser Operation verwendete der Operateur eine Jamshidinadel (Hohlnadel), die nicht in der Navigationssoftware hinterlegt war. Um jedoch mit diesem Instrument navigationsgestützt in den Wirbelkörper Th12 eindringen zu können, musste diese erst in einem speziellen Verfahren kalibriert werden.
Dazu wurde ein SureTrak ${ }^{\circledR}$-II-System benötigt. Dieses System ist ein Metallrahmen, an den auch Einweg-Reflektormarker gesteckt werden. Dieses System kann mit jedem beliebigem Instrument verbunden werden.

Die Navigationssoftware ermöglicht, dass beispielsweise die Jamshidinadel mit einer virtuellen Spitze verlängert werden kann, um so den idealen Eintrittspunkt durch die Haut in den Wirbelkörper zu planen. Ferner kann auch die Höhe oder Breite eines Wirbelkörpers gemessen werden.

Aufgrund der Messungen am gebrochenen Th12 entschied der Operateur bei der linken Hälfte des Wirbelkörpers eine Kyphoplastie und bei der rechten Hälfte des Wirbelkörpers, wegen der begrenzten Platzverhältnisse bei torquiertem und völlig deformiertem Th12, eine Vertebroplastie durchzuführen.

Nach Lokalisation der Eintrittspunkte, zunächst des linken Pedikels Th12, erfolgte eine kleine Hautinzision mit dem Skalpell. Dann navigiertes ( $\rightarrow$ Abb. 8 ) und röntgenkontrolliertes Eingehen mit der Jamshidinadel in den linken Th12-Pedikel. Über die Jamshidinadel wurde ein stumpfer Kirschnerdraht in den Wirbelkörper geschoben, die Jamshidinadel entfernt und ein Arbeitstrokar für die Kyphoplastie über den Kirschnerdraht in den Wirbelkörper vorgeschoben. Das gleiche Vorgehen erfolgte auch auf der rechten Seite des Th12.
Mit einem speziellen Präzisionsbohrer bohrte der Operateur einen Kanal für den 15 Millimeter langen Kyphoplastie-Ballonkatheter in die Spongiosa des Wirbelkörpers.

Anschließend wurde die Arbeitstrokarlage in zwei Ebenen im 2-D-Modus kontrolliert. Dann wurde der Kyphoplastieballon über den linken Arbeitstrokar eingeführt und mit der Insufflation begonnen ( $\rightarrow$ Abb. 9). Links konnten 1,5 Milliliter bis zu einem Druck von 250 PSI insuffliert werden. Im Weiteren erfolgten eine Desufflation des Ballons und die Entfernung des Ballonkatheters aus dem Wirbelkörper.

Nun konnte der Knochenzement angemischt werden. Nachdem der Knochenzement die richtige Konsistenz hatte, wurden links 2,5 Milliliter und rechts 1,5 Milliliter mit Zementapplikatoren in den Wirbelkörper eingebracht.

Abschließend wurde ein kollimierter 3-D-Scan nur im Bereich des Th12 durchgeführt. Zementaustritte nach intraspinal oder extravertebral waren nicht nachweis$\operatorname{bar}(\rightarrow$ Abb. 10).

Zuletzt wurden noch die Arbeitstrokare und die Wirbelsäulenreferenzklemme entfernt, die Röntgenkontrolle beziehungsweise Röntgendokumentation in mehreren Ebenen ( $\rightarrow$ Abb. 11, 12), die Wundspülung, die Hautdesinfektion und die Hautnaht sowie das Anlegen eines Pflasterverbands und die Entlagerung der Patientin auf den Rücken durchgeführt.

\section{(11)}

Auch im Nachhinein erwies sich die Entscheidung, eine hochauflösende 3-D-Rekonstruktion der Anatomie in Verbindung mit der Navigation zu nutzen, als die sicherste und erfolgreichste Methode. Es ist erleichternd und zufriedenstellend, wenn Patienten in schwierigen Situationen geholfen werden kann und technische Möglichkeiten beziehungsweise innovative Medizingeräte vorhanden sind, um Patienten mit einem geringstmöglichen Risiko therapieren zu können.

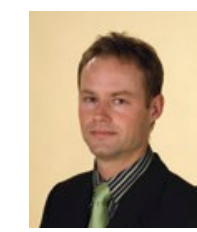

Autor

\section{Andreas Draxinger}

Diplom-Pflegewirt (FH), Gesundheits- und

Krankenpfleger, Stellvertretende OP-

Leitung, Orthopädische Fachklinik

Schwarzach

E-Mail: adraxinger@t-online.de

\section{Literatur}

1 Wikipedia. Kyphose. Im Internet: https://de.wikipedia.org/wiki/Kyphose; Stand 27.07.2016

\section{Bibliografie}

DOI 10.1055/s-0042-113707

Im OP 2016; 6: 248-252

(c) Georg Thieme Verlag KG

Stuttgart · New York · ISSN 1611-7905 